\title{
Factores que determinan la alta satisfacción del usuario con la asistencia hospitalaria
}

\section{Factors associated with patient satisfaction with hospital care}

\author{
T. Dierssen-Sotos ${ }^{1,2}$, P. Rodríguez-Cundín ${ }^{3}$, M. Robles-García ${ }^{4}$, V. Brugos-Llamazares ${ }^{1}$, \\ I. Gómez-Acebo ${ }^{1,2}$, J. Llorca ${ }^{1,2}$
}

\section{RESUMEN}

Objetivo. Identificar la influencia sobre la alta satisfacción de factores sociodemográficos, asistenciales y de comunicación.

Material y Métodos. Estudio de corte transversal mediante encuesta telefónica a pacientes dados de alta durante el mes de septiembre de 2006. Instrumento de medida: cuestionario SERQVHOS. Como medida de fuerza de asociación se ha utilizado la odds ratio (OR) y su intervalo de confianza (IC) al 95\%. Para el ajuste por factores de confusión se ha utilizado el análisis de regresión logística.

Resultados. La tasa de respuesta fue del 80,9\%. Al evaluar la influencia de características sociodemográficas, se observa que los usuarios con nacionalidad no perteneciente a la Unión Europea mostraron mayor satisfacción que los españoles (OR=3,01 IC 95\%: 1,40-3,51), y los entrevistados con estudios primarios resultaron más satisfechos que los universitarios (OR=2,85 IC 95\% [1,88-4,35]). En cuanto a factores asistenciales, en los pacientes con reingresos por la misma categoría diagnóstica mayor se observó una menor prevalencia de alta satisfacción, con una OR de 0,67 y significación limítrofe. Por último, respecto a la comunicación, los tres aspectos más asociados a la alta satisfacción fueron la identificación correcta del personal (OR: 6,22 IC $95 \%[3,52-10,97])$, recibir información suficiente sobre el estado de salud (OR: 3,83 IC 95\% [1,94-7,57]) y recibir información sobre el lugar y horario de información médica (OR: 2,60 IC95\% [1,60-4,22]).

Conclusiones. La comunicación incide favorablemente en la percepción final de la asistencia lo que justifica que se continúe trabajando en su mejora en los centros sanitarios de nuestra comunidad.

Palabras clave. Satisfacción del paciente. Gestión de la calidad. Hospital. Comunicación. Factores socioeconómicos.

\begin{abstract}
Objective. To identify the influence of sociodemographic, care and communication factors on patient satisfaction.

Methods. Cross-sectional study by means of a telephonic survey of patients discharged during September, 2006. Instrument for measuring satisfaction: SERQVHOS questionnaire. Statistical analysis: we used odds ratio (OR) and its 95\% confidence interval (CI); adjustment for confusion factors was performed using multiple logistic regression.
\end{abstract}

Results. Response rate was $80.9 \%$. On evaluating the influence of sociodemographic characteristics, we observed that patients whose nationality did not correspond to the European Union were more satisfied than Spanish patients (OR=3.01; 95\% CI: $1.40-3.51$ ); subjects who had only completed primary studies were more satisfied than those with university studies $(\mathrm{OR}=2.85,95 \% \mathrm{CI}$ : 1.88-4.35). With respect to care factors, patients readmitted because of the same major diagnostic category had lower satisfaction $(\mathrm{OR}=0.67$, bordering statistical significance). Finally, with regard to communication factors, the three aspects most associated with patient satisfaction were: correct health-care personnel identification (OR: 6.22 95\% CI: 3.52-10.97), receiving enough information about his/her state of health (OR: 3.83 95\% CI: 1.94 $7.57)$, and being informed about the place and schedule for medical information (OR: 2.60, 95\% CI: 1.60-4.22).

Conclusions. Communication favourably affects the final perception of care, which justifies the continuity of its improvement in the health centres of our region.

Key words. Patient satisfaction. Quality management. Hospital. Communications. Socioeconomic factors.

\section{An. Sist. Sanit. Navar. 2009; 32 (3): 317-325}

1. Grupo de Medicina Preventiva y Salud Pública. Facultad de Medicina. Universidad de Cantabria. 2. CIBER Epidemiología y Salud Pública (CIBERESP).

3. Servicio de Medicina Preventiva y Salud Pública. Hospital de Sierrallana. Torrelavega.

4. Dirección General de Planificación, Calidad, Ordenación y Formación. Consejería de Sanidad. Junta de Castilla y León.

Recepción 27 de abril de 2009

Aceptación provisional 25 de mayo de 2009

Aceptación definitiva 13 de julio de 2009

\section{Correspondencia}

Trinidad Dierssen-Sotos

Facultad de Medicina

Universidad de Cantabria

Avda. Herrera Oria s/n

39011 Santander

E-mail: trinidad.dierssen@unican.es 


\section{INTRODUCCIÓN}

La satisfacción del paciente constituye una medida del resultado de la asistencia sanitaria que ha sido relacionada directamente con la mejora del cumplimiento terapéutico del paciente, del empleo de seguridad social o de la continuidad de cuidados ${ }^{1,2}$.

La metodología de aproximación a la satisfacción del usuario más utilizada en nuestro medio son las encuestas ${ }^{3}$ que habitualmente evalúan este constructo a partir de dimensiones como el trato/amabilidad, la información, la calidad del resultado, la competencia técnica y la accesibilidad, incorporando además una medida global de satisfacción con el conjunto de la asistencia recibida ${ }^{4,5}$. Entre los instrumentos más utilizados ${ }^{6}$ destacan el Patient Judgements of Hospital Quality ${ }^{7}$, la Encuesta de Experiencias del Paciente ${ }^{8}$, el Consumer Assessment of Health Plans ${ }^{9}$, el Picker Patient Experience Questionnaire ${ }^{10} \mathrm{y}$, en España, el SERVQHOS ${ }^{11}$, una adaptación al sector sanitario de la encuesta SERVQUAL ${ }^{12}$.

En las últimas décadas se han publicado numerosos trabajos relacionando la satisfacción tanto con factores sociodemográficos como con aspectos relativos a la asistencia sanitaria ${ }^{13-19}$. Los primeros, constituyen características intrínsecas y, por lo tanto, no susceptibles de modificación, aunque sí de control. En el metanálisis de Hall y Dorn ${ }^{13}$ el peso de los factores sociodemográficos fue escaso, pudiéndose considerar predictores menores de la satisfacción. Los segundos, las características asistenciales, permiten identificar aspectos en su mayoría modificables por la organización.

El objetivo de este trabajo ha sido conocer la influencia que tienen sobre la alta satisfacción con la asistencia tres grupos de factores: características del paciente, de la asistencia recibida y la información proporcionada durante el ingreso.

\section{MATERIAL Y MÉTODOS}

Se ha desarrollado un estudio de corte transversal mediante entrevista telefó- nica asistida por ordenador. El trabajo de campo fue realizado por teleoperadores previamente formados para explicar el propósito de la investigación, formular adecuadamente las preguntas y dar solución a cuantas dudas e incidencias que pudieran surgir. Las encuestas se realizaron durante el periodo comprendido entre el 10 y el 23 de octubre de 2006.

\section{Población de estudio}

Pacientes dados de alta hospitalaria durante el mes de septiembre de 2006 en los tres hospitales pertenecientes al Servicio Cántabro de Salud: Hospital Universitario Marqués de Valdecilla (HUMV) que cuenta con un promedio anual de 907 camas en funcionamiento, unos 36.700 ingresos y es el hospital de referencia en Cantabria; el Hospital Comarcal Sierrallana que cuenta con 200 camas en funcionamiento y más de 10.600 ingresos anuales y el Hospital Comarcal de Laredo que cuenta con unas 100 camas y unos 5.500 ingresos anuales.

La predeterminación del tamaño muestral se realizó en base a un error de muestreo de $\pm 5 \%$ en cada estrato de cada centro, con un nivel de confianza del 95\% y una prevalencia esperada del 85\%. Se llevó a cabo un muestreo estratificado en 4 grupos: tres correspondientes a los tres servicios que en cada centro presentaron un mayor volumen asistencial y el cuarto correspondiente al resto de servicios evaluados conjuntamente. En el HUMV se añadió un estrato representativo de las altas en la Unidad de Alta Resolución Hospitalaria (UARH).

Para mejorar la tasa de respuesta se remitió, los días previos a la llamada, una carta de presentación incluyendo el nombre del entrevistador, el nombre de la institución, cómo se obtuvo el nombre del participante, un breve enunciado del objetivo del estudio y una estimación de la duración de la llamada. Para disminuir un posible sesgo de recuerdo la encuesta se realizó mediante entrevista telefónica durante los 30 días posteriores al alta. 


\section{Cuestionario de evaluación}

El instrumento de medida de la satisfacción utilizado fue el cuestionario SERQVHOS adaptado para su aplicación telefónica. Este cuestionario ha sido previamente validado por sus autores en nuestro ámbito sanitario, obteniendo valores de Alfa de Cronbach de $0,96^{20}$. Consta de 3 partes, un primer bloque compuesto por 16 preguntas que recogen aspectos clave de la atención valorados de 1 (la calidad de la asistencia ha sido mucho peor de lo esperado) a 5 (mucho mejor), una encuesta adicional que consta de 10 preguntas dicotómicas sobre distintos aspectos de la asistencia y una pregunta directa sobre el nivel de satisfacción global tras el alta hospitalaria. La satisfacción global se evaluó modificando la escala de medida de la encuesta original (variable ordinal con 5 categorías) a numérica (puntuando la asistencia de 0 a 10). Esta modificación se realizó con el objetivo de poder identificar la alta satisfacción de acuerdo a la distribución de la variable en nuestro estudio.

La variable dependiente utilizada fue la satisfacción global dicotomizada en base al percentil 70 de las puntuaciones que se situó en 9 puntos. Las variables de exposición se han agrupado en:

1. Factores sociodemográficos (que incluyen: edad, sexo, estado de salud percibida tras el alta, lugar de residencia y nacionalidad).

2. Características asistenciales (el hospital de ingreso, el número de procedimientos realizado durante el ingreso, ingresos previos desde enero de 2006, si la asistencia evaluada era un reingreso precoz y si se trató de un reingreso por la misma categoría diagnóstica mayor).
3. Factores relacionados con la comunicación durante el ingreso (identificación del personal, información sobre su estado de salud, información sobre lugar y horario de información médica, etc.).

La base de datos del cuestionario se fusionó con la base del conjunto mínimo de datos (CMBD) al alta del paciente, de la que se extrajeron los datos sobre estancia media, procedimientos realizados e intervención quirúrgica.

\section{Análisis estadístico}

Para la comparación de puntuaciones se ha utilizado la prueba $t$ de Student o la U de Mann Whitney para datos no paramétricos.

Para cuantificar el grado de asociación entre las variables de exposición a estudio y la satisfacción se ha utilizado la odds ratio (OR) y su intervalo de confianza al 95\% (IC 95\%). El análisis ajustado se ha realizado mediante regresión logística no condicional.

\section{Aspectos éticos}

Previa a la realización de la encuesta se realizó una llamada para explicar los motivos del estudio y solicitar la aceptación de los usuarios seleccionados.

\section{RESULTADOS}

Durante el periodo de estudio se realizaron 1.268 encuestas telefónicas lo que supone una tasa de respuesta del $80,92 \%$. La tabla 1 resume las principales características de la población de estudio. 
Tabla 1. Características de la población de estudio.

\begin{tabular}{llcc}
\hline & & Frecuencia & Porcentaje \\
\hline Sexo & Hombre & 582 & 45,9 \\
& Mujer & 686 & 54,1 \\
\hline Grupo de edad & Menor de 16 años & 51 & 4,0 \\
& De 16 a 25 años & 84 & 6,6 \\
& De 26 a 35 años & 185 & 14,6 \\
& De 36 a 45 años & 121 & 9,5 \\
& De 46 a 55 años & 113 & 8,9 \\
& De 56 a 65 años & 157 & 12,4 \\
& De 66 a 75 años & 213 & 16,8 \\
& Más de 75 años & 344 & 27,1 \\
\hline Hospital & HUMV & 670 & 52,8 \\
& H. de Sierrallana & 307 & 24,2 \\
\hline Hiempo transcurrido desde el ingreso a la entrevista (días) & 35 & 22,9 \\
\hline
\end{tabular}

Tabla 2. Características sociodemográficas y satisfacción global con la asistencia.

\begin{tabular}{|c|c|c|c|c|}
\hline & 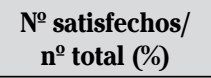 & $\begin{array}{l}\text { OR crudo } \\
\text { [IC 95\%] }\end{array}$ & $\begin{array}{l}\text { OR ajustado } \\
\text { [IC 95\%] }\end{array}$ & $\mathbf{p}$ \\
\hline \multicolumn{5}{|l|}{ Nacionalidad } \\
\hline Unión Europea & $2 / 6(33,3 \%)$ & $1,21[0,2-6,62]$ & $1,46[0,26-8,24]$ & 0,668 \\
\hline Otras nacionalidades & $14 / 30(46,7 \%)$ & $2,11[1,02-4,37]$ & $3,01[1,40-3,51]$ & 0,005 \\
\hline Española & $357 / 1219(29,3 \%)$ & 1 & $1^{\mathrm{a}}$ & \\
\hline \multicolumn{5}{|l|}{ Residencia } \\
\hline Otras & $27 / 76(35,5 \%)$ & $1,32[0,81-21,46]$ & $1,35[0,83-2,20]$ & 0,234 \\
\hline Cantabria & $344 / 1167(29,5 \%)$ & 1 & $1^{\mathrm{b}}$ & \\
\hline \multicolumn{5}{|l|}{ Salud percibida } \\
\hline Muy buena & $81 / 215(37,7 \%)$ & $1,71[0,65-4,52]$ & $1,89[0,70-5,15]$ & 0,210 \\
\hline Buena & $143 / 503(28,4 \%)$ & $1,13[0,44-2,91]$ & $1,17[0,42-2,96]$ & 0,825 \\
\hline Ni buena ni mala & $96 / 323(29,7 \%)$ & $1,20[0,43-3,13]$ & $1,10[0,41-2,93]$ & 0,855 \\
\hline Mala & $41 / 117(35,0 \%)$ & $1,53[0,56-4,18]$ & $1,37[0,49-3,87]$ & 0,547 \\
\hline Muy mala & $6 / 23(26,1 \%)$ & 1 & $1^{\mathrm{a}}$ & \\
\hline \multicolumn{5}{|l|}{ Nivel de estudios } \\
\hline Menos que primarios & $48 / 156(30,8 \%)$ & $1,82[1,10-3,00]$ & $1,73[1,03-2,93]$ & 0,04 \\
\hline Primarios & $200 / 479(41,8 \%)$ & $2,93[1,94-4,42]$ & $2,85[1,88-4,35]$ & $<0,001$ \\
\hline Secundarios & $86 / 329(26,1 \%)$ & $1,45[0,93-2,25]$ & $1,45[0,93-2,25]$ & 0,104 \\
\hline Universitarios & $35 / 178(19,7 \%)$ & 1 & $1^{\mathrm{b}}$ & \\
\hline
\end{tabular}

a: ajustado por edad, sexo y nivel de estudios.

b: ajustado por edad y sexo. 
Al evaluar la influencia de las características sociodemográficas del paciente sobre la alta satisfacción (Tabla 2) los dos factores más fuertemente asociados fueron la nacionalidad, que triplicó la odds ratio en el caso de usuarios procedentes de países no pertenecientes a la UE (OR: 3,01 IC 95\% [1,40-3,51]) y el nivel de estudios que mostró una relación inversa con la alta satisfacción con solo un $19,7 \%$ de usuarios altamente satisfechos entre los entrevistados con estudios universitarios frente al $41,8 \%$ entre los que presentaron estudios primarios, presentando una odds ratio de 2,85 en relación al nivel superior de estudios. Otras características analizadas fueron el lugar de residencia (dicotomizado en residentes en Cantabria y resto) que presentó un mayor porcentaje de alta satisfacción en el grupo de usuarios de otras CCAA (35,5\% frente a $29,5 \% \mathrm{p}=0,264$ ) aunque la diferencia no fue estadísticamente significativa y la percepción del estado de salud que cuando fue valorado como muy bueno aumentó un $89 \%$ la odds ratio de alta satisfacción aunque sin alcanzar la significación estadística. No se observó tendencia lineal en la relación entre salud percibida y alta satisfacción.
En cuanto a la influencia del tipo de asistencia recibida (Tabla 3), se observó en primer lugar que los pacientes ingresados en el HUMV y en Sierrallana presentaron un porcentaje de pacientes muy satisfechos en torno al $32 \%$, claramente por encima del observado cuando la hospitalización se realizó en Laredo (23,6\%), aunque tras el ajuste por potenciales factores de confusión se perdió la significación de esta asociación. Para aproximarnos a la experiencia previa del usuario en el Servicio Cántabro de Salud se valoró la presencia de ingresos previos en el mismo centro a lo largo del año 2006 utilizando tres tipos de variables: presencia de reingresos de enero a septiembre, si la última asistencia había sido un reingreso precoz (en los 30 días después del alta) y si el último ingreso había sido por la misma categoría diagnóstica mayor (CDM) que un ingreso previo en el periodo enero-septiembre. Solo se encontró relación en este último caso, mostrando los pacientes ingresados por la misma CDM un menor porcentaje de alta satisfacción (24,6\% frente a $30,5 \%)$ con una odds ratio de 0,67 y significación limítrofe.

Tabla 3. Consumo de recursos sanitarios y satisfacción global con la asistencia.

\begin{tabular}{|c|c|c|c|c|}
\hline & №satisfechos/ $\mathbf{n}^{\mathbf{0}}$ total (\%) & OR crudo [IC 95\%] & OR ajustado [IC 95\%] & $\mathbf{P}$ \\
\hline \multicolumn{5}{|l|}{ Hospital } \\
\hline HUMV $^{c}$ & $172 / 576(30,6 \%)$ & $1,42[1,03-1,97]$ & $1,34[0,95-1,88]$ & 0,095 \\
\hline H. Sierrallana & $96 / 305(31,5 \%)$ & $1,49[1,03-2,14]$ & $1,31[0,89-1,92]$ & 0,169 \\
\hline H. Comarcal de Laredo & $68 / 288(23,6 \%)$ & 1 & $1^{\mathrm{a}}$ & \\
\hline \multicolumn{5}{|c|}{ № de procedimientos realizados } \\
\hline Resto & $366 / 1219(30,0 \%)$ & $0,64[0,29-1,42]$ & $0,72[0,32-1,64]$ & 0,435 \\
\hline 6 ó más & $8 / 37(21,6 \%)$ & 1 & $1^{a}$ & \\
\hline \multicolumn{5}{|l|}{ Intervención quirúrgica } \\
\hline Sí & $148 / 475(31,2 \%)$ & $1,11[0,86-1,42]$ & $1,05[0,81-1,36]$ & 0,728 \\
\hline No & $217 / 749(29,0 \%)$ & 1 & $1^{\mathrm{a}}$ & \\
\hline \multicolumn{5}{|l|}{ Duración del ingreso } \\
\hline$\leq 9$ días & $300 / 984(30,5 \%)$ & $1,16[0,86-1,58]$ & $1,12[0,80-1,55]$ & 0,495 \\
\hline$>9$ días & $71 / 259(27,4 \%)$ & 1 & $1^{\mathrm{a}}$ & \\
\hline \multicolumn{5}{|l|}{ Reingresos } \\
\hline Sí & $89 / 303(29,4 \%)$ & $0,97[0,73-1,29]$ & $0,89[0,66-1,20]$ & 0,442 \\
\hline No & $285 / 953(29,9 \%)$ & 1 & $1^{\text {a }}$ & \\
\hline \multicolumn{5}{|l|}{ Reingreso precoz } \\
\hline Sí & $32 / 99(32,3 \%)$ & $1,13[0,73-1,76]$ & $1,07[0,70-1,69]$ & 0,777 \\
\hline No & $339 / 1144(29,6 \%)$ & 1 & $1^{a}$ & \\
\hline \multicolumn{5}{|l|}{ Reingreso por la misma CDM } \\
\hline Sí & $34 / 138(24,6 \%)$ & $0,58[0,22-1,12]$ & $0,67[0,44-1,03]$ & 0,068 \\
\hline No & $337 / 1105(30,5 \%)$ & 1 & $1^{\mathrm{a}}$ & \\
\hline
\end{tabular}

a: ajustado por edad, sexo y nivel de estudios. 
No se observaron diferencias relevantes entre el nivel de alta satisfacción y otras variables relacionadas con el consumo de recursos como la intervención quirúrgica durante el ingreso, el número global de procedimientos realizados o la duración del ingreso.

Por último, los aspectos relativos a la comunicación o información durante el ingreso se valoraron a partir de distintas preguntas relacionadas con la información clínica, la información general y la remisión de informes al alta (Tabla 4). Los tres aspectos que mostraron mayor relación con la alta satisfacción fueron la identificación correcta del personal, que multiplicó por 6 la odds de alta satisfacción (OR: 6,22 IC 95\% [3,52-10,97]); haber recibido información suficiente en relación al estado de salud, que la multiplicó por 3,83 (OR: 3,83 IC 95\% [1,94-7,57]) y haber recibido información sobre el lugar y horario de información médica, que la duplicó (OR: 2,60 IC 95\% [1,60$4,22])$. Otros aspectos relativos a la información como conocer el nombre del médico o la supervisora de enfermería o recibir al alta informa médico o de enfermería no mostraron relación con la alta satisfacción.

Tabla 4. Información recibida y satisfacción global con la asistencia

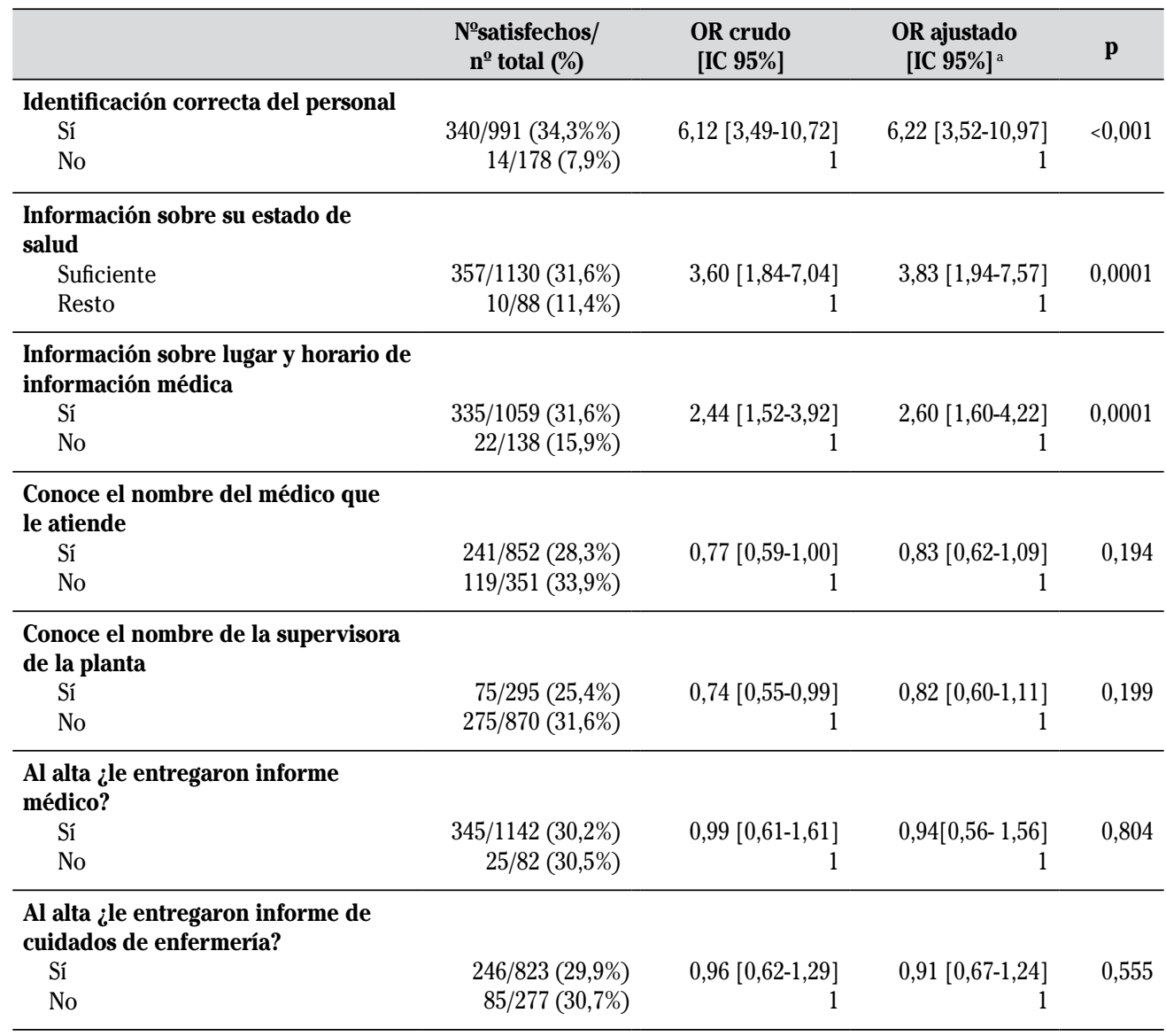

\footnotetext{
a: ajustado por edad, sexo, nivel de estudios y nacionalidad.
} 


\section{DISCUSIÓN}

Este trabajo analiza la influencia de tres grandes grupos de variables sobre la alta satisfacción (factores sociodemográficos, características asistenciales y comunicación), mostrando como resultado más relevante que la valoración positiva de aspectos relacionados con la información o comunicación durante el ingreso, en sus distintas vertientes (información clínica, información general, identificación profesional), se encuentra fuertemente asociada a la alta satisfacción con la asistencia. Estos resultados son congruentes con los aportados por otros autores que observan también un mayor grado de satisfacción global asociado a la mejora de la información en distintos ámbitos asistenciales ${ }^{15-18}$ o a la inversa una relación entre la insatisfacción y la mala valoración de la información proporcionada por el médico ${ }^{19}$ Sin embargo, otras variables relacionadas con la comunicación como el conocer el nombre del médico o de la enfermera que constituyen predictores de calidad percibida en otros estudios ${ }^{21}$, no muestran relación con la satisfacción en este trabajo. Por otra parte, destaca también en nuestro estudio la influencia de algunos factores sociodemográficos como son la nacionalidad del usuario o su nivel de estudios. Los usuarios procedentes de países no pertenecientes a la UE presentaron un mayor porcentaje de alta satisfacción. Otros estudios realizados en el sistema sanitario americano ${ }^{14,22-25}$ han encontrado relación entre la raza y la satisfacción, presentando los usuarios de minorías étnicas una menor satisfacción. Estas discrepancias probablemente se deban a las diferencias entre sistemas sanitarios en aspectos directamente relacionados con la satisfacción como la accesibilidad a la asistencia. Aunque no disponemos de información sobre el país de origen, si predominaran usuarios procedentes de países con menor nivel socioeconómico y por tanto peor cobertura sanitaria, este hecho facilitaría una percepción de nuestro sistema sanitario más positiva.

Por otra parte, la relación entre la satisfacción y el nivel de estudios presenta resultados discrepantes en la literatura médica. En este trabajo los usuarios con nivel de estudios primarios presentaron la mayor proporción de satisfechos mientras que entre los universitarios se observó el nivel más bajo, en consonancia con lo obtenido por Hall y Dornan ${ }^{13}$ y en un estudio previo realizado en nuestro ámbito ${ }^{26}$. Estos resultados contrastan con los aportados por otros autores que encuentran mayor satisfacción en pacientes con mayor nivel de estudios ${ }^{27-29}$. En cuanto al estado de salud, distintos trabajos publicados ${ }^{14,27,30-31}$ muestran una relación directa entre la salud percibida y la satisfacción aumentando ésta a medida que mejora la percepción de la propia salud. En nuestro estudio, el escaso número de efectivos por categoría dificulta la obtención de resultados estadísticamente significativos.

Por último, en este trabajo los aspectos asistenciales evaluados han sido los que presentaron menor asociación con la alta satisfacción. Únicamente el reingreso por la misma CDM se identifica como un factor que incide negativamente en la alta satisfacción. La falta de asociación con la presencia de reingresos previos es consistente con los resultados publicados por Candlish y col${ }^{32}$ en un estudio realizado en ancianos con insuficiencia cardiaca congestiva. Nuestro resultado podría indicar que la asistencia sanitaria se percibe peor cuando no puede resolver el problema de salud del paciente, de modo similar a los resultados aportados por Kristen y $\mathrm{col}^{33}$ que, al evaluar la satisfacción tras histerectomía, encuentran que el reingreso por complicaciones tras el alta es un factor fuertemente asociado a insatisfacción. En cuanto al tipo de hospital en el que se desarrolla la asistencia, el de menor tamaño y complejidad presentó los niveles más bajos de usuarios altamente satisfechos. Estos resultados contrastan con la hipótesis expuesta por Kane $\mathrm{y} \mathrm{col}^{31}$ que sugieren que los centros con mayores niveles de complejidad asistencial obtendrían peores resultados a expensas de una mayor proporción de pacientes con mala salud y en la misma línea con los resultados obtenidos por Young y $\mathrm{col}^{14}$ que observaron 
una relación inversa entre el nivel de satisfacción y el tamaño del hospital. Otros factores asistenciales como la duración del ingreso (relacionada previamente con menores niveles de satisfacción en las dimensiones de confort, y limpieza no han presentado relación con la satisfacción en nuestro estudio.

En resumen, puesto que la principal utilidad de la evaluación de la satisfacción radica en la identificación de aspectos de mejora, a la vista de nuestros resultados, consideramos prioritario incidir en la mejora de la información proporcionada durante el ingreso en los centros sanitarios de nuestra comunidad, puesto que se trata de un factor modificable, dependiente de la organización y fuertemente asociado a la satisfacción.

\section{BIBLIOGRAFÍA}

1. Bell R, KRIVICH J, Boyd MS. Charting patient satisfaction. Mark Health Serv 1997; 17: 22-29.

2. Tasso K, Behar-Horenstein LS, Aumiller A, Gamble K, Grimaudo N, Guin P et al. Assesing patient satisfaction and quality of care trough observation interview. Hospital topics: researchs and perspectives on healthcare 2002; 80: 4-10.

3. Granado de la Orden S, Rodríguez Rieiro C, Olmedo LuCErón MC, Chacón García A, Vigil Escribano D, Rodríguez Pérez P. Diseño y validación de un cuestionario para evaluar la satisfacción de los pacientes atendidos en las consultas externas de un hospital de Madrid en 2006. Rev Esp Salud Pública 2007; 81: 637-645.

4. Hall J, Dornan M. What patients like about their medical care and how often they are asked: a meta-analysis of the satisfaction literature. Soc Sci Med 1988; 27: 935-939.

5. Hall JA, Dornan MC. Meta-analysis of satisfaction with medical care: description of research domain and analysis of overall satisfaction levels. Soc Sci Med 1988; 27: 637-644.

6. Asenjo Velasco C, Colomer Revuelta C, Espiga López I, García Izaguirre C, López Rodríguez R, Peiró Pérez R. Aspectos básicos de gestión de calidad en el ámbito sanitario. En: Informe Anual SNS 2005 Disponible en http://www. msc.es/organizacion/sns/informeAnualSNS/ docs/2005/Cap2AspeBasicGestCalidadAmbitoSanitario.pdf.
7. Meterko M, Nelson E, Rubin H, Batalden P, BERWICK D, HaYs R. Ware Patients` judgements of hospital quality: report of a pilot study. Med Care 1990; 29 № 9: S1-S56.

8. Zastowny TR, Stratmann WC, Adams EH, Fox ML. Patient satisfaction and experience with health services and quality of care. Qual Manag Health Care 1995; 3: 50-61.

9. Hays RD, Shaul JA, Williams VSL, Lubalin JS, HaRRIS-KoJETIN LD, SwEeny SF et al. Psychometric properties of the CAHPS 1.0 survey measures. Med Care 1999; 37 (Suppl): MS22-31.

10. Jenkinson C, Coulter A, Bruster S, Picker Patient Experience Questionnaire: development and validation using data from in-patient surveys in five countries. Int J Qual Health Care 2002; 14: 353-358.

11. Mira JJ, Aranaz J, Rodríguez-Marín J, Buil JA, CAstell M, Vitaller J. SERVQHOS: un cuestionario para evaluar la calidad percibida de la asistencia hospitalaria. Med Preventiva 1998; IV: 12-18.

12. Parasuraman A, Zeithaml V, Berry L. SERVQUAL: a multipleitem scale for measuring consumer perceptions of service quality. J Retailing 1988; 64: 12-40.

13. Hall JA, Dornan MC. Patient sociodemographic characteristics as predictors of satisfaction with medical care: a meta-analysis [published erratum appears in Soc Sci Med 1990; 30 (12): following 1368]. Soc Sci Med 1990; 30: 811-818.

14. Young GJ, Meterko M, Desai KR. Patient satisfaction with hospital care: effects of demographic and institutional characteristics. Med Care 2000; 38: 325-334.

15. Billings K, Newland Franzco H, Selva Franzco D. Improving patient satisfaction through information provision. Clin Exp Ophtalmology 2007; 35: 439-447.

16. Nerney MP, Chin MH, Jin L, KarRison TG, WaLter J, Mulliken R et al. Factors associated with older patients`satisfaction with care in an inner-city emergency departament. Ann Emerg Med 2001; 38: 140-145.

17. Vidal O, Romero JM, Ginestà C, Badiella L, VALENTINI M, ESPERTA JJ et al. Factores asociados con la satisfacción en el cuidado y la asistencia en el departamento de cirugía de urgencias de los pacientes mayores de 65 años. Cir Esp 2008; 83: 260-265.

18. Castillo L, Dougnac A. Vicente I, Muñoz V, Rojas V. Predictors of the level of patient satisfaction in a university hospital. Rev Med Chile 2007; 135: 696-701. 
19. MirA JJ. "No estar satisfecho» no equivale a "estar insatisfecho", Rev Clin Esp 2006; 206: 208-212.

20. Mira JJ, Aranaz J, Lorenzo S, Rodríguez-Marín J, Moyano S. Evolución de la calidad percibida por los pacientes en dos hospitales públicos. Psicothema 2001; 13: 581-585.

21. Mira JJ, Bull JÁ, Aranaz J, Vitaller J, Lorenzo $\mathrm{S}$, IGnacio E, Rodríguez-Marín J et al ¿Qué opinan los pacientes de los hospitales públicos? Análisis de los niveles de calidad percibida en cinco hospitales. Gac Sanit 2000; 14: 291293.

22. BARR DA. Using the appropriate method to test for perceived differences in care. J Gen Intern Med 2004; 19: 937-943.

23. Crow R, Gage H, Hampson S, Hart J, Kimber A, Storey L et al. The measurement of satisfaction with healthcare: implications for practice from a systematic review of the literature. Health Technol Assess 2002; 6: 1-244.

24. Weech-Maldonado R, Morales LS, Elliott M, Spritzer K, Marshall G, Hays RD. Race/ethnicity, language, and patients` assessments of care in medicaid managed care. Health Serv Res 2003; 38: 789-808.

25. Campbell JL, Ramsay J, Green J. Age, gender, socioeconomic, and ethnic differences in patients`assessments of primary care. Qual Health Care 2001; 10: 90-95.

26. Robles-García M, Dierssen-Sotos T, Llorca-Díaz J, Díaz-Mendi AR, Herrera-Carral P. En busca de la excelencia: el perfil del paciente «supersatisfecho". Rev Calidad Asistencial 2004; 19 : 34-37.

27. XIAO H, BARBER JP. The effect of perceived health status on patient satisfaction. Value Health 2008; 11: 719-725.

28. Becker G, Newsom E. Socioeconomic status and dissatisfaction with healthcare among chronically ill African Americans. Am J Public Health 2003; 93: 742-748.

29. Maderuelo JA, Haro AM, Pérez F, Cercas LJ, Valentín AB, Morán E. Satisfacción de las mujeres con el seguimiento del embarazo. Diferencias entre los dispositivos asistenciales. Gac Sanit 2006; 20: 31-39.

30. Covinsky KE, Rosenthal GE, Chren MM, Justice AC, Fortinsky RH, Palmer RM et al. The relationship between health status changes and patient satisfaction in older hospitalized medical patients. J Gen Intern Med 1998; 13 : 223-229.

31. Kane RL, Maciejewsi M, Finch M. The relationship of patient satisfaction with care and clinical outcomes. Med Care 1997; 35: 714-730.

32. Candlish P, Watts P, Redman S, Whyte P, Lowe J. Elderly patients with heart failure: a study of satisfaction with care and quality of life. Int $\mathrm{J}$ Qual Health Care 1998; 10: 141-146.

33. KJerulfF KH, Rhodes JC, Langenberg PW, Harvey LA. Patients satisfaction with results of hysterectomy. Am J Obstet Gynecol 2000; 183: 1440-1447. 
